\title{
Conflictos en torno al acceso al agua potable en el barrio Felix U. Camet, provincia de Buenos Aires: de la problematización
} \section{a la acción}

\author{
Leonardo Lupi \\ lupi@mdp.edu.ar \\ (iD) orcid.org/0000-0002-6292-6496

\section{María Belén Ceretta} \\ Consejo Nacional de Investigaciones \\ Científicas y Técnicas-Universidad \\ Nacional de Mar del Plata, Argentina. \\ mbceretta@mdp.edu.ar \\ (iD) orcid.org/0000-0003-2577-0506
}

RECEPCIÓN: 27/04/21

ACEPTACIÓN FINAL: 08/06/21

\section{Resumen}

El presente artículo describe y reflexiona sobre el trabajo del Grupo Aguas, Universidad Nacional de Mar del Plata, en el barrio Félix U. Camet (partido de General Pueyrredón, Buenos Aires, Argentina) entre los años 2008-2012, donde se desarrolló un proceso de organización en torno a la problemática del consumo de agua no potable. El objetivo del artículo es analizar el accionar desde la perspectiva de la Extensión Crítica y la Investigación-Acción Participativa a través de entrevistas y revisión de documentación. Así pues, queda de manifiesto que el trabajo del Grupo Aguas fue fundamental en el establecimiento de los procesos subyacentes a la problemática y en la consolidación de un grupo de vecinos que demandaron acciones al Estado. Por otro lado, se vislumbra que el conocimiento científico se resignifica cuando es apropiado de forma colectiva para dar a conocer las necesidades del territorio y contribuir en promover acciones tendientes a lograr la transformación de la problemática.

Palabras clave: agua potable; contaminación; conflictos sociales; extensión crítica; Investigación Acción Participativa.

\author{
Gonzalo Martín Peruzzaro \\ luzbaiong@gmail.com \\ (iD) orcid.org/0000-0003-2604-8267

\section{Joaquín Pinciroli} \\ joacopinciroli@gmail.com \\ (iD) orcid.org/0000-0002-8148-0557
}

Universidad Nacional de Mar del Plata, Argentina.

Conflicts around access to drinking water in the Felix U. Camet, Buenos Aires province neighborhood: from problematization to action

\section{Abstract}

This article describes and reflects on the work of Grupo Aguas, Mar del Plata National University, in Félix U. Camet neighborhood (Ptdo. Gral. Pueyrredón, Buenos Aires, Argentina) between 2008-2012, where an organization process was developed around the problem of non-potable water consumption. The objective of the article is to analyze the actions from the perspective of Critical Extension and Participatory Action Research, through interviews and documentation review. Thus, it is clear that the action of the Grupo Aguas was fundamental in establishing the processes underlying the problem, and in the consolidation of a group of neighbors who demanded actions from the State. On the other hand, it is seen that scientific knowledge is resignified when it is collectively appropriate to make known the needs of the territory and contribute to promoting actions aimed at achieving the transformation of the problem.

Keywords: drinking water; pollution; social conflicts; critical extension; Participatory Action Research.
Ambiente y extensión universitaria /

\author{
Conflitos em torno do acesso à \\ água potável no bairro Felix U. \\ Camet, província de Buenos Aires: \\ da problematização à ação
}

\section{Resumo}

Este artigo descreve e reflete sobre o trabalho do Grupo Águas do bairro Félix U. Camet (Ptdo. Gral. Pueyrredón, Buenos Aires, Argentina) entre 2008-2012, onde se desenvolveu um processo de organização em torno do problema de consumo de água não potável. O objetivo do artigo é analisar a ação na perspectiva da Extensão Crítica e da Pesquisa-Ação Participativa, por meio de entrevistas e revisão documental. Assim, ficou claro que o trabalho do Grupo Águas foi fundamental para estabelecer os processos subjacentes ao problema e para a consolidação de um grupo de vizinhos que exigia ações do Estado. Além disso, percebe-se que o conhecimento científico se ressignifica quando é coletivamente adequado para fazer saber as necessidades do território e contribuir para a promoção de ações que visem a transformação do problema social e ambiental.

Palavras-chave: água potável; contaminação; conflitos sociais; extensão crítica; Pesquisa-Ação Participativa.

Para citación de este artículo: Lupi, L.; Ceretta, M. B.; Peruzzaro, G. M. y Pinciroli, J. (2021). Conflictos en torno al acceso al agua potable en el barrio Felix U. Camet, provincia de Buenos Aires: de la problematización a la acción. +E: Revista de Extensión Universitaria, 11(14), e0010. doi: 10.14409/extension.2021.14.Ene-Jun.e0010 


\section{Introducción}

En Argentina, el acceso a fuentes de agua potable no es universal ni equitativo y se calcula actualmente que el $20 \%$ de la población no lo tiene, en tanto que los pobladores más afectados son los de barrios populares y los de zonas periurbanas y rurales. ${ }^{1}$ A comienzos de 2008, a raíz de la detección de una serie de casos relacionados con problemas gastrointestinales, la trabajadora social de la posta sanitaria del barrio Félix U. Camet (FUC) del partido de General Pueyrredón (PGP, Buenos Aires, Argentina) contactó a integrantes del Grupo Aguas (GA) ante la sospecha de que los problemas de salud se vinculaban con el consumo de agua contaminada. El GA, grupo de extensión de la Facultad de Ciencias Exactas y Naturales de la Universidad Nacional de Mar del Plata (FCEyN-UNMdP) integrado por estudiantes, graduados y docentes de diversas disciplinas, trabaja en la evaluación de la calidad del agua consumida en distintos barrios periurbanos y rurales sin servicio de agua potable y contribuye a problematizar este derecho vulnerado. A través del trabajo realizado, se apuesta a generar procesos de organización en pos de transformar esta realidad.

Tras el contacto inicial de la trabajadora social, durante los siguientes 3 años se realizaron muestreos en el barrio para analizar la calidad del agua en las perforaciones domiciliarias, según los parámetros microbiológicos establecidos en el Código Alimentario Argentino, talleres de formación sobre calidad del agua subterránea y devolución de resultados de los muestreos. El propósito inicial del trabajo era dar a conocer la calidad de agua consumida por los habitantes. De esta manera, todo aquel que solicitaba la toma de una muestra era relevado, exceptuando aquellos que tenían un vecino contiguo que había sido analizado previamente. Así fue que se promovió, a través de un proceso de organización, la apropiación de los resultados por parte de la comunidad con el fin de contribuir a la construcción de una demanda colectiva por agua potable.

Diversos interrogantes surgen con relación a cómo impactó la acción del GA en el barrio FUC entre los años 2008 y 2012. Para analizar el devenir de este proceso se recurrió a registros audiovisuales, notas periodísticas, informes técnicos y otra información de archivo. También se efectuaron cuatro ${ }^{2}$ entrevistas en profundidad, semiestructuradas y de manera individual, a vecinos que participaron activamente de los hechos, quienes contribuyeron en la revisión de este artículo.

Con la información recabada se definieron dos ejes a desarrollar: i) a nivel barrial, describir y analizar el proceso de organización que se dio a partir del accionar del GA y en torno a la problemática de acceso a agua potable; ii) a nivel municipal, describir y analizar el conflicto que emerge entre vecinos y representantes institucionales/del Estado en cuanto al acceso y provisión de agua potable. Para abordar el análisis de los ejes planteados se utilizaron los siguientes indicadores: información disponible respecto de la potabilidad de los pozos, tipo de comunicación y profundidad de la misma entre vecinos y el GA, participación de los vecinos, forma de organización de estos antes y después del trabajo del GA, nivel de conocimiento de la problemática en el territorio y acciones desarrolladas para transformarla. El objetivo final de la sistematización de esta experiencia es definir si efectivamente la intervención del

1) https://www.argentina.gob.ar/obras-publicas/hidricas/agua-potable-y-saneamiento-0

2) Se les denominará Vecino 1, Vecino 2, Vecino 3 y Vecino 4 para preservar su identidad, en concordancia con lo acordado durante las entrevistas realizadas durante el año 2020. 
GA en el barrio FUC constituyó una praxis extensionista enmarcada en la Extensión Crítica (EC) (Medina y Tommasino, 2018) y la Investigación-Acción Participativa (IAP) (Fals Borda, 1997), entendiendo a la EC como un tipo de práctica (acción) colectiva tendiente a transformar las problemáticas del territorio a partir de su conocimiento. En ese sentido, se reconoce que el trabajo del GA y su posterior evaluación colectiva constituyen un proceso posible de enmarcar en la IAP.

\section{Del problema a la causa colectiva}

Al inicio del trabajo del GA en el año 2009, FUC no contaba con servicios de cloacas ni red de agua, por lo que los vecinos se abastecían mediante perforaciones de agua domiciliarias. Si bien, previo a la intervención del GA, pocos vecinos habían hecho analizar el agua de su perforación de manera particular y formalizado reclamos, estas acciones aisladas no llegaron a ser significativas. Por otro lado, la inminente instalación de la Nueva Planta de Tratamiento de Residuos Cloacales (NPTRC) contigua a FUC, así como las instalaciones deficitarias del Jardín de Infantes $\mathrm{N}^{\circ} 29$, formaban parte de las preocupaciones más frecuentes. A la luz de los primeros resultados que daban cuenta de que cerca del $90 \%$ de las muestras eran no potables (Álvarez et al., 2010), comenzaron a nuclearse vecinos interesados en la problemática.

De esta manera empezaron a realizarse reuniones periódicas, cuya frecuencia fue en aumento conforme se desarrollaron los muestreos hasta que llegaron a ser semanales. En los primeros encuentros organizados por el GA se evidenció que la contaminación del agua que consumían los vecinos era una problemática generalizada. En los talleres se daban a conocer los resultados obtenidos de los muestreos y se discutían las posibles causas de la contaminación y medidas paliativas para su prevención (Figura 1). Al finalizar la etapa de relevamiento, se habían analizado 165 muestras de agua, de las cuales un 87 \% resultó no potable, particularmente por exceso de bacterias coliformes (Bader et al., 2011). En este contexto, el GA promovía la discusión y búsqueda de acciones con el conjunto de la comunidad afectada, de manera que tanto la problemática como la solución eran de carácter colectivo.

Figura 1. Esquema de trabajo del GA en el barrio FUC

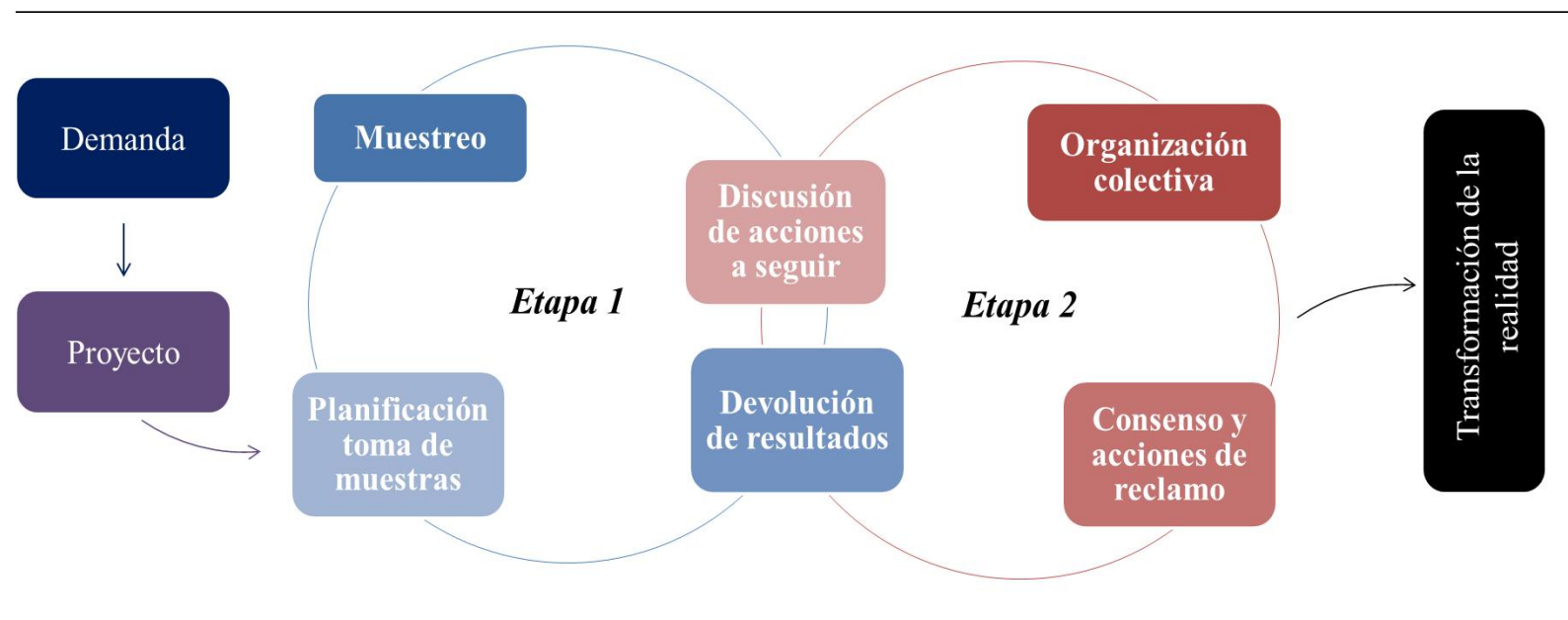

Fuente: elaboración propia, 2020. 
Los lugares donde se llevaron a cabo las reuniones fueron variando, inicialmente fueron en la plaza central del barrio, luego en la Sociedad de Fomento (SF), la parroquia, y con posterioridad en casas de vecinos. Sin embargo, después de un tiempo los vecinos acordaron retornar a la plaza ya que la consideraban un "terreno neutral", como destacó uno de los entrevistados. Las reuniones o asambleas eran abiertas a todo el barrio. Estos encuentros eran espacios de discusión, intercambio de ideas, reflexión y toma de decisiones, donde todos tenían la posibilidad de hablar, emitir su opinión y disentir. El GA tuvo un papel central en la organización de las asambleas

"fue muy importante el reglarnos de alguna manera como asamblea, el poder organizarnos, y eso dependió en buena medida de la cuestión como más metódica que traían los chicos del GA, (...) porque si no éramos un montón de gente con buenas intenciones". (Entrevista a Vecino 1, noviembre de 2020)

Conforme se pedía la palabra se establecía el orden de oradores y el sistema de toma de decisiones era por mayoría, levantando la mano. Como expresó otro entrevistado: "Podíamos expresarnos tranquilamente y exponer nuestras ideas (...). Todo lo que se hizo se hizo con acuerdo de los vecinos" (Entrevista a Vecino 3, noviembre de 2020). En este ámbito, los integrantes del GA no solo asistían a las asambleas sino que además eran considerados como un vecino más, podían opinar, proponer y votar al igual que el resto.

Llegado este punto del desarrollo de la experiencia fue posible evidenciar al menos dos aspectos centrales que denotan el posicionamiento del GA respecto de la extensión universitaria. Por una parte, la problemática emergió de los actores territoriales, quienes detectaron algún aspecto conflictivo de su vida cotidiana o de la dinámica del barrio y organizaron acciones de manera colectiva para su resolución. Por otra parte, el contacto con el GA da cuenta de la trayectoria y pertinencia del trabajo específico que realiza pero, a su vez, de la necesidad de conocer las determinaciones de la problemática para abordarla con la mayor rigurosidad. La posibilidad de conocer la realidad que se pretendía transformar, a través de los resultados de los relevamientos, constituyó para los vecinos un hecho fundamental. Esto denota la potencialidad del conocimiento científico cuando es apropiado por sus protagonistas y a la extensión universitaria como la herramienta que unifica a distintos sectores de trabajadores en un objetivo común (Freire, 1984).

Sea bajo la denominación de reuniones o asambleas abiertas, lo que importa aquí es el método participativo y horizontal para la construcción del problema y el debate sistemático que promueve la aprehensión de las determinaciones del mismo por parte de los sujetos que disponen su tiempo, cuerpo y recursos para enfrentarlo. Como se menciona en una de las entrevistas:

"los chicos [del Grupo Aguas] tienen su ideología y obviamente había quedada plasmado en el tipo de actividad que hacen, pero creo que siempre se consensuó con Vecinos Autoconvocados (VA), y eso hay que agradecerles, que aunque ellos no hubieran tomado esas decisiones respetaron las decisiones de VA. (...) fue la democracia más pura que llegué a conocer en la República Argentina". (Entrevista a Vecino 2, noviembre de 2020) 
A lo largo de toda esta sección se puso de manifiesto el proceso que se llevó adelante para poder dar con la resolución de un conflicto en el territorio. Esto también incluye la manera en que el saber científico estuvo implicado en el mismo, donde los actores involucrados fundamentaron lo que ocurre en su barrio con evidencia concreta, sin deslegitimar el saber popular previo. Es aquí donde se aborda la problemática desde las bases, donde se reúnen y toman decisiones por mayoría y en conjunto. Este proceso territorializado, desde el abordaje de la problemática hasta su resolución, busca inscribirse en la línea de la Extensión Crítica. Como puede observarse según los registros mencionados anteriormente, el GA toma como referencia esta propuesta a la hora de llevar a cabo su práctica extensionista. A través de talleres y análisis se abordó la problemática y acompañó a los vecinos en las reuniones, también se contribuyó en la formación de la asamblea y se participó en la toma de decisiones.

A su vez, gracias al grado de organización alcanzado, que implicó la creación de comisiones, y a las reflexiones que surgieron durante las asambleas, los vecinos avanzaron sobre otras problemáticas que tenían lugar en el territorio, como la construcción del Jardín de Infantes № 29 y el Centro de Atención Primaria de la Salud FUC (Cáneva et al., 2011). Los entrevistados coinciden en que los logros obtenidos tienen que ver con el proceso de organización, donde el GA tuvo un rol preponderante en la conformación de VA: "el GA fue motorizador de esa primera organización, (...) después es como una bola de nieve que va creciendo" (Entrevista a Vecino 1, noviembre de 2020). Concretamente, la extensión universitaria se constituye en el vínculo entre distintos actores populares por el cual es posible transformar una problemática de la realidad a partir de asumirse como sujetos históricos conscientes.

Cuando el saber científico se pone en práctica y logra develar aspectos de la realidad y, a su vez, se llevan a cabo diferentes acciones para la resolución del mismo, la ciencia cobra sentido. Los vecinos encarnan el movimiento por el cual un aspecto particular se vuelve colectivo en tanto comparten un territorio que es entendido como el espacio de las relaciones sociales que los constituyen como tales, donde se dirimen intereses contrapuestos y donde, por lo tanto, la acción colectiva por regirse con conocimiento de causa es una acción política.

Los dos aspectos que dan cuenta de que estamos frente a un proceso enmarcado en la EC son: que toda acción política contiene un momento de reflexión en el que se vuelve consciente una circunstancia de la realidad que aparece como problemática, y que esa misma acción será efectiva si es apropiada de manera colectiva por sujetos que definen una forma participativa de llevar adelante el conflicto. El aspecto central radicó en la organización y visibilización pública de la problemática de modo colectivo. Desde ese lugar se potenciaron los reclamos y demandas por parte de un grupo de vecinos que tomó conciencia de que el derecho al agua potable estaba siendo vulnerado.

Ahora bien, en todo conflicto aparecen fuerzas sociales contrapuestas que representan o personifican intereses particulares. En lo que sigue delimitaremos las diferentes respuestas de actores institucionales a la demanda por agua potable, la caracterización de cada uno de ellos realizada por los vecinos de FUC, y pondremos en discusión la idea de Estado en tanto sujeto que aparece como externo, mediando el conflicto, y las características que lo determinan como un Estado "presente o ausente" o "que incluye o excluye" al tomar para sí la exigencia de derechos elementales por parte de los vecinos. 


\section{Las ambigüedades y el Estado, el posicionamiento frente a la temática del agua}

En el PGP, la encargada de la prestación del servicio de agua potable es la empresa municipal de servicios sanitarios Obras Sanitarias Sociedad de Estado (OSSE). Desde su creación en 1984 tiene a su cargo "la prestación, administración, explotación, mantenimiento, control, ampliación, renovación, construcción, estudios, investigación y aplicación de nuevas tecnologías de los servicios de provisión de agua potable". ${ }^{3}$ En este sentido, cuando se hizo pública la problemática del consumo de agua contaminada, los representantes institucionales de OSSE, la Municipalidad y la SF del barrio se vieron compelidos a exponer su posición y tomar medidas formales.

En los testimonios de los entrevistados aparecieron de modo contundente los cambios de posición, en particular de OSSE y la SF, respecto de la posibilidad de abastecimiento de agua potable, los resultados de calidad de agua generados por el GA y la promoción de acciones comunitarias.

Previo a la llegada del GA, los reclamos realizados por vecinos de manera particular a OSSE fueron ignorados sistemáticamente por la institución. Hacia el año 2010, luego de surgir los primeros resultados del consumo de agua no potable que daban sustento al reclamo, OSSE promovió el descrédito de la información, produciéndose como no existente. Ante la insistencia de los vecinos, OSSE argumentó la imposibilidad técnico-económica de las obras de agua de red, considerando que la baja densidad poblacional del barrio no justificaba los costos de construcción de un acueducto desde la zona norte, y por tal motivo no se llevaría agua potable. La confianza de la información generada por el GA fue puesta a prueba mediante distintas situaciones. Algunos vecinos mandaron a corroborar sus resultados particulares a laboratorios privados con el fin de acreditar el resultado de potabilidad. Otros notaron las contradicciones por parte de OSSE a lo largo del proceso de organización vecinal:

"Ellos [el GA] en principio empezaron con un muestreo. Después Obras Sanitarias se opuso al muestreo, como sacándole validez al estudio que realizaron los chicos. Oh, casualidad, después de todo esto OSSE empezó un muestreo particular de ellos y el clorado de los pozos". (Entrevista a Vecino 1, noviembre de 2020)

Por último, el fortalecimiento del vínculo y confianza de los VA hacia GA se consolidó con una metodología de trabajo participativa, colaborativa y honesta en cuanto a las intenciones políticas y a la horizontalidad en la toma de decisiones, como se mencionó en el apartado previo.

A medida que el grupo de VA se consolidaba y junto con ello las acciones públicas colectivas, OSSE comenzó a intervenir con el fin de abordar la problemática y contener la movilización respondiendo en parte a la demanda. La empresa colocó un tanque de gran capacidad en la plaza central del barrio y posteriormente proveyó a la SF con un tanque cisterna para abastecimiento ambulante de agua potable. Sin embargo, el tanque cisterna fue utilizado por la SF para obras privadas en construcción, lo cual ameritó una denuncia por parte de VA. ${ }^{4}$

Con relación a la SF, se observa que intervino de dos maneras distintas según la instancia del desarrollo de la problemática y organización vecinal. Inicialmente puso a disposición el 
espacio físico de la institución para la realización de talleres, lo que le permitía mejorar su imagen en el barrio porque trataba la problemática y difundía sus gestiones en la materia durante los años previos a la llegada del GA. Sin embargo, a medida que se consolidaba el proceso comunitario, la SF boicoteó las acciones de diferentes formas: negó la posibilidad de uso de las instalaciones o intervino para que no se prestaran otras, como la parroquia del barrio, y también obstruyó los avances conseguidos mediante la organización. Incluso, la SF llegó a interponerse en el desarrollo de actividades mediante el amedrentamiento y violencia física durante una jornada de reclamo por agua potable: "la Sociedad de Fomento no era la mejor, no estaba abierta la participación de los vecinos, de hecho nos trabaron un montón de cosas que se venían intentando hacer" (Entrevista a Vecino 1, noviembre de 2020), situación que puede constatarse en una nueva denuncia por parte de los VA. ${ }^{5}$

En este marco, el hostigamiento de las instituciones del Estado trascendió las fronteras de lo barrial, puesto que los integrantes del proyecto fueron citados por las autoridades de la FCEyN durante el año 2011, a solicitud de autoridades de OSSE, para dar explicaciones sobre las acciones del proyecto. Esta tensión se profundizó en 2012, cuando el GA fue incitado a cesar las actividades de laboratorio destinadas al trabajo de extensión, lo que originó una denuncia de la situación ante la comunidad universitaria y solidaridad de los VA por medio de un breve informe de desempeño del GA para ser presentando ante el Consejo Académico de la Facultad. ${ }^{6}$

Poco después de la denuncia pública del consumo de agua contaminada ante el Concejo Deliberante del Municipio del PGP mediante la Banca $25,{ }^{7}$ se implementó a través de ENOHSA el Plan Agua + Trabajo, ${ }^{8}$ creado por el gobierno nacional con el fin de llevar los servicios de agua potable y cloacas a las áreas más vulneradas. De esta manera es posible observar cómo la política asistencial es la forma que toma la acción por parte del Estado con el fin de dar respuesta a los reclamos de los vecinos organizados. El agua para abastecer la red fue extraída de una perforación de OSSE realizada para dicho fin en el mismo barrio, lo que evidencia que las soluciones técnicas emergen si existe la voluntad política de resolver el problema del agua contaminada.

Sin embargo, la ejecución de las obras tuvo varias dificultades y limitaciones vinculadas a malversaciones por parte de la SF y situaciones inherentes al trabajo precario en el marco de las cooperativas: "Yo veía que había como intereses creados, intereses monetarios [de la SF], no intereses comunes". Y se agrega: "Vino una cooperativa de otro lado, no cumplieron con los tiempos, no le pagaban a la gente, se robaban las cosas. Terminó yéndose..." (Entrevista a Vecino 3, noviembre de 2020).

De este modo se completa una parte del trabajo inicial con intervención de OSSE, aunque en la actualidad los reclamos continúan, ya que las obras representan solo un $30 \%$ de la superficie del barrio. 
Figura 2. Línea de tiempo

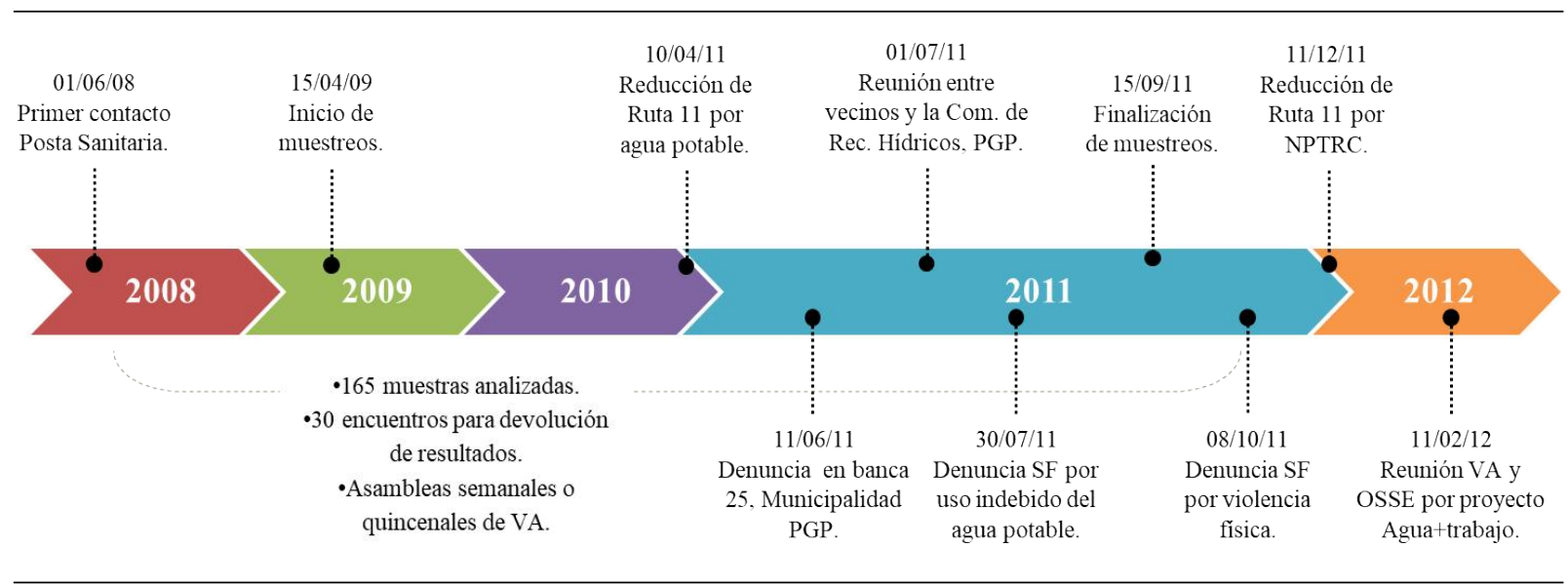

Fuente: elaboración propia 2021

Se advierte un cambio claro de posicionamiento de ambas instituciones en el inicio de las actividades del GA y durante el transcurso de la disputa. El Estado, frente a una problemática donde se puso en juego inicialmente la credibilidad del GA, captó para sí la lucha por el agua potable mediante una política asistencial. A través de sus diferentes instituciones impuso sus formas de "resolver" el problema, incluso de forma violenta. En este sentido, como menciona Núñez (2009), se pudo ver cómo la SF entra en juego como una institución de legitimación del poder y no meramente como una institución vecinal a la cual recurrir para resolver un conflicto, lo que se verifica en las acciones ya mencionadas.

Por otra parte, en lo que respecta a OSSE, denota que no es más que una institución que vehiculiza la demanda por parte de los vecinos acorde a los intereses propios y del municipio. Incluso se puso en evidencia en el momento en que solo realizaron las obras en una fracción minoritaria del barrio, no en la totalidad. Se puede decir que OSSE juega un papel en el que posee el poder de decisión, por sobre la demanda exigida por los vecinos, niega el suministro, al menos en parte, y demuestra así el papel de las instituciones del Estado y su personal político en la reproducción social. Por su parte, el gobierno nacional implementó un programa de obras públicas para acceso al agua, evento donde queda de manifiesto, una vez más, que el Estado toma para sí el reclamo de los vecinos por la red de agua y cloacas: de manera precaria, descentralizada, y sin participación de los vecinos.

\section{Recapitulando...}

Los vecinos, a lo largo del conflicto, pasaron de acciones de reclamo formales y empleo de vías institucionales a otras de mayor impacto en términos de respuesta de actores políticos y de visibilidad mediática. En un inicio, los reclamos se centraron en enviar cartas documento solicitando información acerca de las futuras obras de agua y cloaca para el barrio y la construcción de dichos servicios. Estas instancias se llevaron a cabo tanto en las oficinas de OSSE como en el municipio. Asimismo, con el fin de acelerar dichas gestiones se mantuvieron encuentros informales con concejales de partidos diversos y distintas autoridades de 
OSSE. Sin embargo, ante sucesivas dilaciones y estrategias disuasivas que obstaculizaron una respuesta favorable, los vecinos optaron por realizar una acción de alto impacto mediático: un corte parcial o reducción de la Ruta Nacional № 11 (RN11), a pesar de que esta medida fue rechazada de plano por los mismos vecinos al inicio del proceso. ${ }^{9}$

Las medidas que involucran un compromiso corporal y riesgo elevado durante la ejecución del reclamo en un marco que roza la ilegalidad, como los cortes de ruta, permiten un mayor despliegue de energía, entendida como grado de disposición para la lucha (Nieto-SISMO, 2019). Esta clase de protesta y visibilización marcó un antes y un después en el proceso, luego de la cual los servicios sanitarios de FUC formarían parte de la agenda gubernamental, reclamo que posteriormente se formalizó con otra medida de implicación corporal media, como lo fue la presentación de los resultados de calidad de agua en la Banca $25 .{ }^{10}$ Los entrevistados manifestaron en forma reiterada que el corte parcial de la RN11 fue una acción con un elevado grado de efectividad en la visibilización y producción del conflicto. Este aprendizaje se hizo evidente en la consecución de reiteradas acciones mediáticas (Figura 2), un nuevo retorno a la ruta meses después para el reclamo de información y audiencia pública por la NPTRC, ${ }^{11}$ así como en el reclamo de otras demandas (como la construcción del edificio del Jardín que se concretó en 2013). ${ }^{12}$

\section{Reflexiones finales}

Hay varios aspectos que resultan relevantes a la hora analizar la acción del GA en este proceso. La construcción de un vínculo fuerte entre vecinos e integrantes del GA no hubiera sido posible sin rigurosidad técnica y una práctica honesta mediada por espacios participativos y democráticos. Dicha acción fue fundamental tanto en el establecimiento de los procesos subyacentes a la problemática del consumo de agua contaminada como en la consolidación de un grupo de vecinos, quienes fueron ganando experiencia organizativa e implementando diversas estrategias para la instalación y resolución de las problemáticas. Si bien el aporte técnico-conceptual fue realizado por el GA y apropiado por los VA —reflejado con claridad en la presentación en la Banca 25-, es importante mencionar que ello no estableció una jerarquía en la toma de decisiones sino que disolvió la figura del "universitario" o "científico" en los espacios asamblearios donde, finalmente, fueron dirimidas las decisiones.

Asimismo, desde una perspectiva técnico-científica, la información producida por el GA no fue valorada en sí por su carácter original o novedoso, sino más bien por las acciones que desencadenó en el ámbito social. La participación de manera auténtica en procesos organizativos implica, a veces, supeditar propuestas o decisiones individuales o de grupos reducidos a aquellas definidas colectivamente. En este sentido, diferencias en la estrategia de visibilización y reclamo por el agua fueron explicitadas y superadas en el marco de toma de decisiones de manera asamblearia y democrática. Esta situación no solo ocurrió en el ámbito barrial sino también hacia el interior del GA, considerando que se generaron instancias de

9) Reducción de calzada RN 11, abril 2011. Félix U. Camet.

10) En la Banca 25, vecinos de Félix U. Camet denunciaron que beben agua contaminada.

11) Protestarán en la ruta para exigir a OSSE información de la planta de pretratamiento de efluentes.

12) El intendente Pulti inauguró el nuevo edificio del Jardín de Infantes Municipal $N^{\circ} 29$ José Luis del Hierro. 
discusión, reflexión y decisión propias, en particular con una medida como la del corte parcial de la RN11, puesto que no todos los integrantes del GA acordaron plenamente con la medida.

Finalmente, mencionamos que, agotadas todas las instancias de reclamos institucionales, los VA no tuvieron más opción que avanzar en acciones de mayor implicación corporal y carácter mediático, tomando conciencia de la efectividad de cada una de ellas. Las acciones generadas permitieron transformar, al menos en parte, la realidad cotidiana vinculada al consumo de agua contaminada y otras preocupaciones vecinales, lo que evidenció la potencia de las acciones colectivas mediadas por el conocimiento científico respecto de los problemas concretos de la realidad y su orientación hacia el reclamo de derechos así como a la construcción de poder popular.

\section{Referencias bibliográficas}

Alvarez, G.; Ambrosio, R.; Arias, L.; Azzone, D.; Bader, A.; Cagliari, Y.; Canneva, L.; Canel, D.; Cañal, V.; Cebuhar, J.; Chierichetti, M.; Commatteo, J.; Cuniolo, A.; Irigoitia, M.; Larreche, M. R.; Lupi, L.; Manzoni, M.; Marte, F.; Naife, A.; Peralta, R. G.; Salomone, M.; Saldain, B.; Sánchez Terreno, C.; Vasini, B.; Zalazar L. (2010) Calidad microbiológica del agua de consumo en el Barrio Félix U. Camet de Mar del Plata. IV Congreso Nacional de Extensión Universitaria y IX Jornadas Nacionales de Extensión Universitaria. Universidad Nacional de Cuyo (UNCUYO).

Bader, A.; Ambrosio, R.; Arias, L.; Canel, D. (2011). Agua microbiológicamente no apta para consumo en el Barrio Félix U Camet de la Ciudad de Mar del Plata. XI Congreso Iberoamericano de Extensión Universitaria. UNL. Canneva, L.; Irigoitia, M.; Lupi, L.; Roldán, R.; Vasini, B.; Zalazar, L. (2011). "GRUPO AGUAS" Junto a los vecinos del Barrio Félix U. Camet de Mar del Plata. XI Congreso Iberoamericano de Extensión Universitaria. UNL. Fals Borda, O. (1997). El problema de cómo investigar la realidad para transformarla por la praxis. Tercer Mundo Editores.

Freire, P. (1984). ¿Extensión o comunicación? La concientización en el medio rural. Siglo XXI Editores. Informe Final (2012). Proyecto de Extensión: "Laboratorio Portátil".

Medina, J. M.; Tommasino, H. (Eds.) (2018). Extensión Crítica: Construcción de una Universidad en contexto. UNR Editora.

Nieto, A.-SISMO (2019). La conflictividad social en clave local. Un ejercicio de ponderación sobre los hechos de rebelión en la Mar del Plata actual (Argentina, 2011-2016). Clivatge. Estudis i testimonis sobre el conflicte i el canvi socials, (7). https://doi.org/10.1344/CLIVATGE2019.7.7

Núñez, A. (2009). Abrir la política urbana y las identidades sociales. Ni empresarios, ni burócratas, ni vecinos: estatalidad profunda y estatalidad extensa. Economía, sociedad y territorio, 9(30). 